\title{
Clinical analysis of 29 cases of nasal mucosal malignant melanoma
}

\author{
HUANXIN YU and GANG LIU
}

\author{
Department of Otorhinolaryngology Head and Neck Surgery, Tianjin Huanhu Hospital, Tianjin 300060, P.R. China
}

Received July 14, 2014; Accepted March 26, 2015

DOI: $10.3892 / 01.2015 .3259$

\begin{abstract}
The present study aimed to investigate the clinical features of nasal mucosa malignant melanoma, including the histopathological features and factors affecting prognosis. A retrospective analysis of the clinical data obtained from the Department of Otolaryngology Head and Neck Surgery, Tianjin Huanhu Hospital (Tianjin, China) between October 1999 and June 2013 was performed using the Kaplan-Meier method. In total, 29 cases of nasal mucosal malignant melanoma were analyzed. The overall 3- and 5-year survival rates were 48.3 and $27.6 \%$, respectively. The study group consisted of 18 males and 11 females, with a median age of 61.5 years. Overall, 19 patients underwent surgery, 28 received radiotherapy and 17 received chemotherapy. The American Joint Committee on Cancer staging system (AJCC) was used to retrospectively stage the tumors. In total, 8 were tumor stage (T)1, 10 were T2, 6 were $\mathrm{T} 3$ and 5 were $\mathrm{T} 4$. The results revealed that the T stage, surgical treatment, location of the tumor and the presence of black pigmentation affected the 5-year survival rate of the patients. By contrast, radiotherapy and chemotherapy had no effect on the overall survival rate. Overall, endoscopic or endoscopic-assisted surgery were the preferred methods of treatment, and histological features, including the presence of tumor melanin pigmentation, affected the prognosis of the patients. This study indicated that the AJCC staging system is able to effectively predict the prognosis of patients with nasal mucosa malignant melanoma.
\end{abstract}

\section{Introduction}

Nasal mucosal malignant melanoma is clinically rare, accounting for $\sim 1 \%$ of all systemic malignant melanomas and $4 \%$ of all malignant nasal tumors (1). Malignant melanoma originates from melanoma cells, which are derived from the neuroectoderm of the ectodermal mucosa (2). Melanoma of the mucosal membrane of the respiratory tract and skin melanoma exhibit different clinical and histopathological characteristics, however,

Correspondence to: Dr Gang Liu, Department of Otorhinolaryngology Head and Neck Surgery, Tianjin Huanhu Hospital, 122 Qixiangtai Road, Tianjin 300060, P.R. China E-mail: liugang60@yahoo.com.cn

Key words: nose neoplasm, nasal mucosa malignant melanoma, combined therapy, prognosis the two have a similar prognosis (2). Nasal mucosal malignant melanoma has high local recurrence and metastasis rates, a poor prognosis and a 5-year survival rate of 20-35\%. Therefore, it can be challenging to achieve a satisfactory curative effect with simple surgical procedures, radiotherapy or chemotherapy (1). The clinical data of 29 patients with nasal mucosal malignant melanoma was obtained from the Department of Otolaryngology Head and Neck Surgery, Tianjin Huanhu Hospital (Tianjin, China) between October 1999 and June 2013, and subsequently analyzed with regard to the clinical features of the disease.

\section{Materials and methods}

Clinical data. In total, 29 patients with nasal mucosa malignant melanoma who had received treatment at the Department of Otolaryngology Head and Neck Surgery, Tianjin Huanhu Hospital between October 1999 and June 2013 were included in the present study. All cases were confirmed for the presence of nasal mucosal malignant melanoma by pathological and immunohistochemical analyses. Patient age, gender, location of tumor, clinical staging, surgical therapy and adjuvant therapy were retrospectively analyzed. The clinical characteristics are shown in Table I. This study was approved by the ethics committee of Tianjin Huanhu Hospital and written informed consent was obtained from all patients.

Histopathological features. All slides were analyzed by three senior pathologists. The pathological features that were investigated included the presence of black pigmentation, the number of cells undergoing mitosis per high-power field, and the presence of bone invasion and necrosis.

Follow-up. Follow-up, with respect to survival and tumor progression, was conducted by phone, letters and visits to the patients.

Statistical analysis. Statistical analyses were performed using SPSS 17.0 software (SPSS, Inc., Chicago, IL, USA). The survival rate was analyzed by the Kaplan-Meier method and log-rank test. Fisher's exact probability was used to analyze the balance between the two groups. $\mathrm{P}<0.05$ was used to indicate a statistically significant difference.

\section{Results}

Patients. The study group included 18 males and 11 females, with a gender ratio of $1.6: 1$. The median age at diagnosis was 


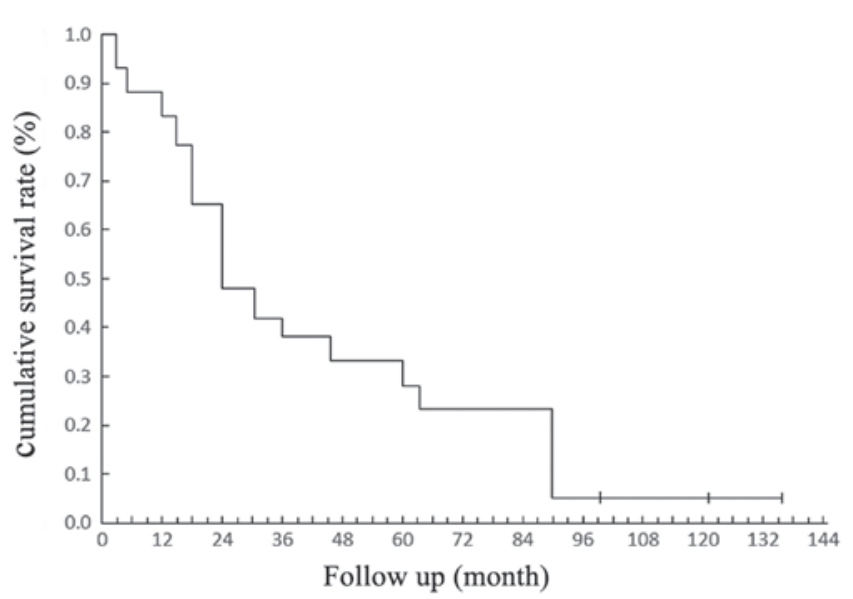

Figure 1. Cumulative survival rate of the 29 patients with nasal mucosal malignant melanoma.

61.5 years (range, 49-73 years). The most common symptoms were nasal hemorrhage (21/29) and nasal obstruction $(15 / 29)$. The initial average duration of symptoms was 33 months. The overall 3 - and 5-year survival rates were $48.3 \%$ and $27.6 \%$, respectively (Fig. 1).

Tumor location and staging. The tumors were located in the nasal septum of 7 patients, the lateral wall of the nasal cavity in 12 patients, the maxillary sinus in 5 patients and the ethmoid sinus in 5 patients. Melanomas in the nasal septum conferred a better prognosis than those in other regions ( $\mathrm{P}=0.008$; Fig. 2). According to the American Joint Committee on Cancer (AJCC) staging system (3), $28 \%$ of patients presented with tumor stage (T) $1,34 \%$ with T2, $21 \%$ with T3 and $17 \%$ with T4. Significant differences were observed in the survival rates of patients with $\mathrm{T} 3+\mathrm{T} 4$ vs. T1 + T2 ( $\mathrm{P}=0.012$; Fig. 3). In total, 5 patients exhibited distant metastasis; 2 presented with brain metastasis, 1 with bone metastasis, 1 with lung metastasis and 1 with liver metastasis.

Treatment. Of the 29 patients, 22 underwent surgical treatment. In total, 15 were treated by endoscopic surgery, 7 were treated by an endoscopic-assisted approach, 5 underwent lateral rhinotomy and 2 underwent resection of the maxilla. The 5-year survival rate of the patients who had undergone surgery was higher than that of those who had not received surgical intervention ( $\mathrm{P}=0.021$; Fig. 4).

In total, 28 patients were treated with radiation therapy at a dose of 30-70 Gy (average, $50 \mathrm{~Gy}$ ). Overall, 17 patients $(58.6 \%)$ received a total radiation dose of $>54$ Gy. The 5-year survival rate of patients administered a radiation dose of $>54$ Gy was not significantly improved $(\mathrm{P}=1.000)$. Chemotherapy was administered to 17 of the patients. However, the results revealed that chemotherapy treatment had no effect on the 5-year survival rate $(\mathrm{P}=0.694$; Table I).

Histopathology and follow-up. The histopathological examination revealed that melanin pigmentation was an important indicator of prognosis $(\mathrm{P}=0.027)$. However, the number of cells undergoing mitosis in each high-power field, and the presence of bone invasion or necrosis, had no statistical significance $(\mathrm{P}>0.05$; Table I).

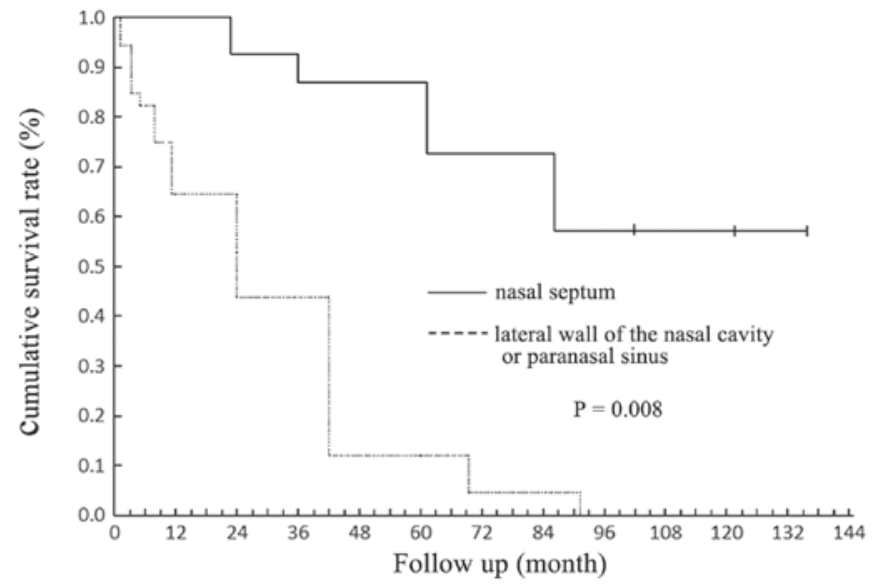

Figure 2. Association between incidence and cumulative survival rate.

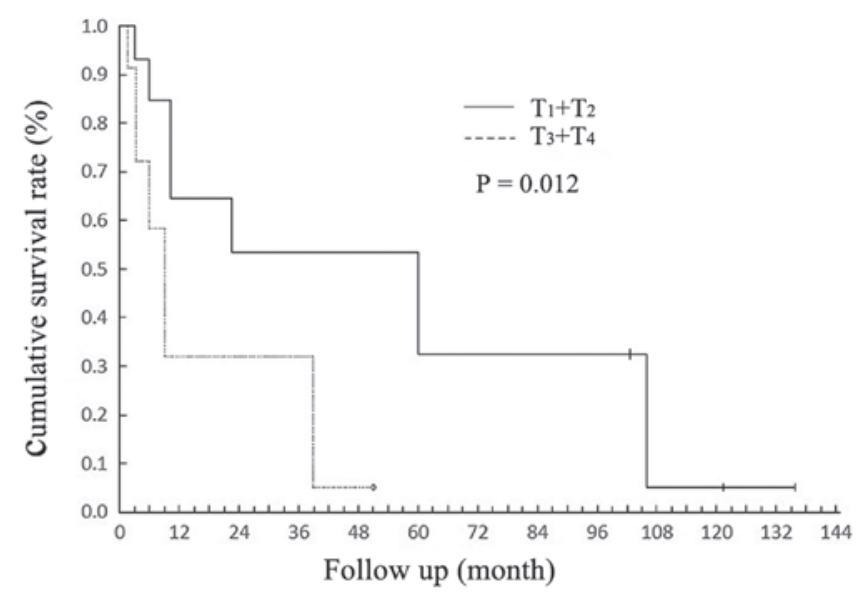

Figure 3. Association between tumor (T) stage and cumulative survival rate.

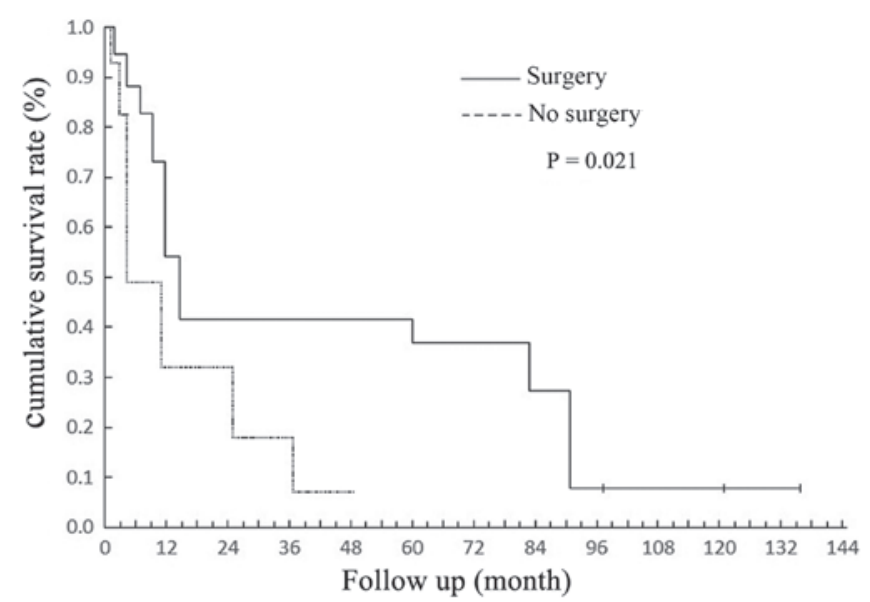

Figure 4. Association between treatment approach and cumulative survival rate.

The patients were followed up for 5-135 months. The median follow-up period was 70.8 months. During follow-up, 15 patients experienced local recurrence, with a recurrence time of 0.8-63 months (average, 18.6 months). Distant metastases occurred in 19 patients, with a transfer time of 2-60 months (average, 12.3 months). 
Table I. Effect of different clinical factors on the survival rate of patients with nasal mucosal malignant melanoma.

\begin{tabular}{|c|c|c|c|}
\hline Factors & $\mathrm{n}$ & 5 -year survival rate, $\%$ & P-value \\
\hline \multicolumn{4}{|l|}{ Gender } \\
\hline Male & 18 & 27.8 & \multirow[t]{2}{*}{1.000} \\
\hline Female & 11 & 27.3 & \\
\hline \multicolumn{4}{|l|}{ Age, years } \\
\hline$\leq 60$ & 10 & 30.0 & \multirow[t]{2}{*}{0.694} \\
\hline$>60$ & 19 & 26.3 & \\
\hline \multicolumn{4}{|l|}{ Tumor location } \\
\hline Nasal septum & 7 & 71.4 & \multirow[t]{2}{*}{0.008} \\
\hline Lateral wall of nasal cavity/paranasal sinus & 22 & 13.6 & \\
\hline \multicolumn{4}{|l|}{ Clinical stage } \\
\hline $\mathrm{T} 1+\mathrm{T} 2$ & 18 & 44.4 & \multirow[t]{2}{*}{0.012} \\
\hline $\mathrm{T} 3+\mathrm{T} 4$ & 11 & 0.0 & \\
\hline \multicolumn{4}{|l|}{ Surgical treatment } \\
\hline Yes & 22 & 36.4 & \multirow[t]{2}{*}{0.021} \\
\hline No & 7 & 0.0 & \\
\hline \multicolumn{4}{|l|}{ Radiotherapy dose, Gy } \\
\hline$\leq 54$ & 11 & 27.3 & \multirow[t]{2}{*}{1.000} \\
\hline$>54$ & 17 & 29.4 & \\
\hline \multicolumn{4}{|l|}{ Chemotherapy } \\
\hline Yes & 17 & 29.4 & \multirow[t]{2}{*}{0.694} \\
\hline No & 12 & 25.0 & \\
\hline \multicolumn{4}{|l|}{ Presence of black pigmentation } \\
\hline Yes & 10 & 0.0 & \multirow[t]{2}{*}{0.027} \\
\hline No & 19 & 42.1 & \\
\hline \multicolumn{4}{|l|}{$>10$ mitoses per high-power field } \\
\hline Yes & 15 & 26.7 & \multirow[t]{2}{*}{1.000} \\
\hline No & 14 & 28.6 & \\
\hline \multicolumn{4}{|l|}{ Evidence of bone invasion } \\
\hline Yes & 7 & 28.6 & \multirow[t]{2}{*}{0.612} \\
\hline No & 22 & 27.3 & \\
\hline \multicolumn{4}{|l|}{ Evidence of necrosis } \\
\hline Yes & 8 & 25.0 & \multirow[t]{2}{*}{1.000} \\
\hline No & 21 & 28.6 & \\
\hline
\end{tabular}

T, tumor.

\section{Discussion}

Malignant melanoma is a malignant tumor of epithelial tissue origin. It has a low incidence rate, often occuring in the skin, but rarely in the mucosa. Primary nasal mucosal malignant melanoma originates from the dendritic melanocytes of the nasal mucosa that develop from the embryonic neural crest, which belongs to the neuroendocrine cell system. Early-stage nasal mucosal malignant melanoma does not exhibit any specific clinical manifestations, which makes early diagnosis challenging and often results in misdiagnosis. The local recurrence and metastasis rates are high, and the prognosis is poor. In recent years, the diagnosis and treatment of this disease has improved due to the widespread use of imaging techniques and endoscopy (1).
A previous study reported that $70 \%$ of malignant melanoma patients were $>60$ years of age, with a male to female ratio of 1.5:1 (4). In the present study, 19 patients were aged 60 years, accounting for $65.5 \%$ of the cohort, and the gender ratio was 1.6:1. The median age at diagnosis was 61.5 years. The results of the present study indicate that gender and age are not important factors affecting nasal mucosal malignant melanoma patients $(\mathrm{P}=1.000$ and $\mathrm{P}=0.694$, respectively).

The lateral wall of the nasal cavity and the nasal septum are common sites for primary nasal mucosal malignant melanoma, accounting for 41.4 and $24.1 \%$ of cases, respectively. The study found that the prognosis of patients with melanoma of the nasal septum was better than that of patients with melanoma of the sinuses or nasal lateral wall $(\mathrm{P}=0.008)$, which was 
similar to the findings of Patel et al (5). This study suggests that the symptoms of melanoma in the nasal septum manifest at an earlier clinical stage than that of melanoma located at other locations, including the sinuses and nasal lateral wall, which subsequently results in earlier diagnosis and treatment.

In 1970, Ballantyne (6) introduced the clinical staging system for melanoma of the skin of the head and neck. This system includes 3 stages: Stage I for localized disease, stage II for regional disease, and stage III for distant metastasis. The prognostic value of this system has been recognized in the literature. According to Ballantyne's clinical staging system, primary nasal mucosal melanoma accounted for $75.9 \%$ of the present study population. Although Ballanyne's clinical stage can reflect the overall survival, the majority of patients in the present study were stage I, whereas the number of stage II and III patients was small (24.1\%), and thus, Ballanyne's clinical stage was not considered an independent prognostic factor. The AJCC staging system provides an even distribution of tumor stages and accurately reflects stage-specific prognostic information, effectively reflecting the 5 year survival rate $(\mathrm{P}=0.012)$ in the present study. Thus, we recommend that the current AJCC sinonasal staging system should be used as the standard staging system for patients with nasal mucosal malignant melanoma. This is consistent with conclusions made by the American Anderson cancer research center study (7). Therefore, we propose that the modern AJCC staging system may be the best melanoma staging system for patients with nasal sinus mucosa.

The surgical treatment for nasal mucosal malignant melanoma is complete resection of the tumor. This traditional surgical approach involves a lateral nasal incision or resection of the maxilla, however, due to the particular anatomical structure of the nasal passage, complete resection of the tumor can be challenging. Surgery is convenient for exposure and resection, but has the disadvantages of a large amount of trauma, bleeding, complications, post-operative scarring and a longer duration of hospitalization (8). With the wide application of nasal endoscopy, endoscopic resection of sinonasal tumors has gradually developed in recent years (9). In the present study, 29 patients underwent surgical treatment. Of those patients, $22(75.9 \%)$ underwent simple nasal endoscopic surgery or endoscopic-assisted surgery. It was revealed that patients who had received surgical treatment exhibited an improved 5-year survival rate $(\mathrm{P}=0.021)$.

The treatment of nasal mucosa malignant melanoma remains controversial. However, comprehensive treatment is widely accepted, in particular, surgery combined with radiotherapy is considered to be the optimum treatment in order to gain local control (10). Nasal mucosal malignant melanoma patients are sensitive to radiation therapy, but the radiation dose remains a point of controversy. Previous data has revealed that adjuvant radiotherapy can reduce the local recurrence rate of tumors, but is unable to improve the overall survival rate for patients. Furthermore, the optimal total dose of radiation and the distribution plan are yet to be determined (11). A previous study found that a high dose (>54 Gy) of irradiation improved the local control rate of tumors and led to a lower recurrence rate (12). However, Krengli et al (13) indicated that large doses of radiotherapy had no clear effect on tumor control or the 5-year survival rate of patients. In the present study, $17(58.6 \%)$ patients received a total dose of $>54 \mathrm{~Gy}$. Despite this, the 5-year survival rate of these patients was not effectively improved $(\mathrm{P}=1.000)$. Analysis showed that, following surgery and/or radiotherapy local tumor control was achieved, however, due to the high rates of local recurrence and metastasis and the poor prognosis, radiation therapy may not improve the overall 5-year survival rate. The present study found that patients receiving chemotherapy did not demonstrate an improved 5-year survival rate $(\mathrm{P}=0.694)$. This may be due to an insensitivity of the malignant melanoma to the chemotherapy drugs.

In cases of head and neck skin malignant melanoma, a risk level can be accurately determined, and the pathological tissue can provide a theoretical basis for the histopathological features (8). However, in cases of nasal mucosal malignant melanoma, there are no clear factors affecting the prognosis pathology. Previous data has established that hyperpigmentation, mitosis, sinus bone invasion and necrosis may affect the prognosis of tumors $(14,15)$. The present study found that the presence of black pigmentation was the only pathological prognostic factor $(\mathrm{P}=0.027)$. By contrast, the number of cells undergoing mitosis, and the absence of sinus bone invasion and necrosis were not associated with patient prognosis.

In summary, although the incidence of nasal mucosal malignant melanoma is relatively low, the prognosis is poor. The present study confirmed that the AJCC sinus grading system can provide an accurate analysis of the prognosis of patients with malignant melanoma of the nasal mucosa. Surgery is the main treatment for such patients, and although the optimal dose of irradiation and its distribution scheme are yet to be determined, auxiliary radiotherapy remains the main or palliative treatment. The presence of black pigmentation may be a pathological prognostic factor. However, due to the small number of cases in the present study, future clinical studies with large sample sizes, randomized grouping and long-term follow-up periods are required in order to validate these findings.

\section{References}

1. Manolidis S and Donald PJ: Malignant mucosal melanoma of the head and neck: review of the literature and report of 14 patients. Cancer 80: 1373-1386, 1997.

2. Prasad ML, Busam KJ, Patel SG, Hoshaw-Woodard S, Shah JP and Huvos AG: Clinicopathologic differences in malignant melanoma arising in oral squamous and sinonasal respiratory mucosa of the upper aerodigestive tract. Arch Pathol Lab Med 127: 997-1002, 2003.

3. Greene FL, Page DL, Fleming ID, Fritz A, Balch CM and Haller DG (eds). AJCC Cancer Staging Manual: 6th edition. Springer-Verlag, New York, NY, pp59-67, 2002.

4. Dauer EH, Lewis JE, Rohlinger AL, Weaver AL and Olsen KD: Sinonasal melanoma: a clinicopathologic review of 61 cases. Otolaryngol Head Neck Surg 138: 347-352, 2008.

5. Patel SG, Prasad ML, Escrig M, et al: Primary mucosal malignant melanoma of the head and neck. Head Neck 24: 247-257, 2002.

6. Ballantyne AJ: Malignant melanoma of the skin of the head and neck. An analysis of 405 cases. Am J Surg 120: 425-431, 1970.

7. Moreno MA, Roberts DB, Kupferman ME, et al: Mucosal melanoma of the nose and paranasal sinuses, a contemporary experience from the M. D. Anderson Cancer Center. Cancer 116: 2215-2223, 2010.

8. Bachar G, Loh KS, O'Sullivan B, et al: Mucosal melanomas of the head and neck: experience of the Princess Margaret Hospital. Head Neck 30: 1325-1331, 2008.

9. Penel N, Mallet Y, Mirabel X, Van JT and Lefebvre JL: Primary mucosal melanoma of head and neck: prognostic value of clear margins. Laryngoscope 116: 993-995, 2006. 
10. Owens JM, Roberts DB and Myers JN: The role of postoperative adjuvant radiation therapy in the treatment of mucosal melanomas of the head and neck region. Arch Otolaryngol Head Neck Surg 129: 864-868, 2003.

11. Temam S, Mamelle G, Marandas P, et al: Postoperative radiotherapy for primary mucosal melanoma of the head and neck. Cancer 103: 313-319, 2005.

12. Wada H, Nemoto K, Ogawa Y, et al: A multi-institutional retrospective analysis of external radiotherapy for mucosal melanoma of the head and neck in Northern Japan. Int J Radiat Oncol Biol Phys 59: 495-500, 2004.

13. Krengli M, Masini L, Kaanders JH, et al: Radiotherapy in the treatment of mucosal melanoma of the upper aerodigestive tract: analysis of 74 cases. A Rare Cancer Network study. Int J Radiat Oncol Biol Phys 65: 751-759, 2006.
14. McLean N, Tighiouart M and Muller S: Primary mucosal melanoma of the head and neck. Comparison of clinical presentation and histopathologic features of oral and sinonasal melanoma. Oral Oncol 44: 1039-1046, 2008.

15. Thompson LD, Wieneke JA and Miettinen M: Sinonasal tract and nasopharyngeal melanomas: a clinicopathologic study of 115 cases with a proposed staging system. Am J Surg Pathol 27: 594-611, 2003 